\title{
TARGET RECOGNITION BASED ON CENTRAL AND LOGARITHMIC CENTRAL IMAGE CHORD TRANSFORMATIONS
}

\author{
Veaceslav Perju*, ORCID ID: 0000-0002-7755-4277 \\ Department of Defense and Security Agency for Military Science and Memory \\ 47 Tighina Str., Chisinau, MD-2001, Republic of Moldova \\ *vlperju@yahoo.com
}

Received: 27.04 .2021

Accepted: 05.06.2021

\begin{abstract}
Object recognition is of great importance for many civil and military applications and supposes the identification and classification of the object in real-time, regardless of spatial position, angular orientation, etc. In the article, the methods of central and logarithmic central image chord transformations are described. The system for invariant object recognition and space parameters determination was developed based on the proposed image chord transformation operations, which is of a multiprocessor functionally distributed architecture, in which various modules implement separate image processing operations, and allows to recognize the objects regardless of their position, scale, or rotation, and to determine the parameters for targeting The model of a data processing flow in the system was elaborated to permit the determination of the signal processing stages which can be realized in parallel. An analytical estimation and analysis of the system's data processing time and throughput is carrying out. The approach of the system's optimization was applied and allows to decrease the total data processing time by 24.5 times and increase the system's throughput by 75 times.
\end{abstract}

Keywords: image, chord, transformation, recognition, system, processor, optical.

\section{Introduction}

Object recognition is of great importance for many civil and military applications and supposes the identification and classification of the object in real-time, regardless of spatial position, angular orientation, etc. Different directions and approaches exist in object recognition.

One of the directions is based on using the support vector machines (SVM). The paper [1] proposes a target detection algorithm based on Bayesian theory and SVM combination which permits to estimate of the probability of partial unknowns with incomplete information. In the article [2] a system is described using a data-dependent Lyapunov function, constructed using the Support Vector Data Description. In article [3] is described an approach based on the calculation of the vectors of spectral and multifractal features which are classified by an SVM.

Many publications were made in the field of neural networks using for invariant pattern recognition. In article [3] a detection algorithm was proposed using a convolutional 
neural network (CNN) and deep learning. The CNN is trained with a large number of positive and negative samples which permits the false detection rate to reduce at maintaining the original detection rate. In article [5] the neural network architectures are proposed to classify the images using the features calculated from the wavelet transform. In article [6] a fusion method was proposed combining the feature obtained by deformable CNN layer to obtain the invariance to diverse scale and shape-changing of an object.

Another direction in invariant pattern recognition is based on the generation of image moment features. In article [7] a deep learning model for object classification using the CNN integrated with a statistical moments layer was proposed. In paper [8] a new set of Fractional-order Chebyshev Moments (FCM) was presented. In the article [9] an effective approach for the computation of Hahn moments was proposed. The gray image is decomposed in a set of binary images - bitplanes, and their moments are computed. In paper [10] a color moment invariant descriptor according to $\mathrm{Hu}$ moment invariants was proposed, which have satisfactory stability for light variations.

Pattern descriptors invariant to rotation, scaling, translation (RST) represent an important direction in pattern recognition. In article [11] the RST invariance is obtained by applying the 1D Fourier-Mellin and discrete Fourier transforms on the radial and angular coordinates of the pattern's Radon image. This algorithm is robust to white and salt-pepper noise. In article [12] a novel descriptor is proposed based on the ring-projection and dualtree complex wavelets which permit the transformation of the pattern from a 2-D image to a 1-D signal. In article [13] the Hough transform realization is proposed in an incoherent optical processor.

The described above directions and approaches in invariant pattern recognition need substantial computational expenditures which in many cases don't permit the realization of real-time in pattern recognition. Also, numerical accuracy does not correspond to the standards.

One of the important directions in automatic pattern recognition is based on the chord functions using due to their properties of invariance to the object position, rotation, and scaling changes. In the article [13] a hybrid optical-digital system for chord functions calculation is described. In article [15] an optical processor that realizes a generalized chord transformation is presented. The wedge-ring detector samples of autocorrelation are shown to be the histograms of the chord distributions. In article [16] are described new methods of image presentation - Central and Logarithmic Central Image Chord Transformations (CIHT and LCIHT).

In this article, the results of the development of the invariant object recognition based on the image chord transformations are presented. Section 2 describes the image transformations - Central and Logarithmic Central Image Chords Transformation (CICT and LCICT). It is shown that the CICT operation makes it possible to achieve invariance to object rotation. In the case of implementation of the LCICT transformation, invariance to changes in the rotation and scale of the object is achieved. In section 3 the structure of an optical processor for image chord transformations based on a matrix of threshold optrons and a photodetector with a radial-circular scan is presented. In section 4 the structure of an optical-electronic system for invariant object recognition based on the proposed image chords transformation operations is presented. In section 5 the model of a data processing flow in the system is given that permits the determination of the stages of signal processing which can be realized in parallel. In section 6 an analytical estimation and analysis of the 
system's data processing time and throughput is carrying out. The data processing stage of maximal processing time was determined. In section 7 the approach of the system's optimization was applied.

\section{Operations of central and logarithmic central image chord transformations}

Let $P(x, y)$ is an initial object and $P_{b}(x, y)$ is the function of the object's image, which is described by the binary external contour, $P_{b}(x, y)=\{0,1\}$ (Figure 1), and $\left(x_{c}, y_{c}\right)$ - coordinates of the center. For every pair of points $\left(x_{i}, y_{i}\right)$ and $\left(x_{k}, y_{k}\right)$ is constructed a chord $A_{i} B_{k}$ passing through the point $\left(\mathrm{x}_{\mathrm{c}}, \mathrm{y}_{\mathrm{c}}\right)$, is determined the angle $\Psi_{\mathrm{ik}}$ between chord and $\mathrm{X}$-axis, and chord's length $\mathrm{R}_{\mathrm{ik}}$ as:

$$
\begin{aligned}
& \psi_{i k}=\operatorname{arctg}\left[\left(\mathrm{y}_{\mathrm{k}}-\mathrm{y}_{\mathrm{i}}\right) /\left(\mathrm{x}_{\mathrm{k}}-\mathrm{x}_{\mathrm{i}}\right)\right] \\
& R_{i k}=\sqrt{\left(\mathrm{x}_{\mathrm{k}}-\mathrm{x}_{\mathrm{i}}\right)^{2}+\left(\mathrm{y}_{\mathrm{k}}-\mathrm{y}_{\mathrm{i}}\right)^{2}}
\end{aligned}
$$

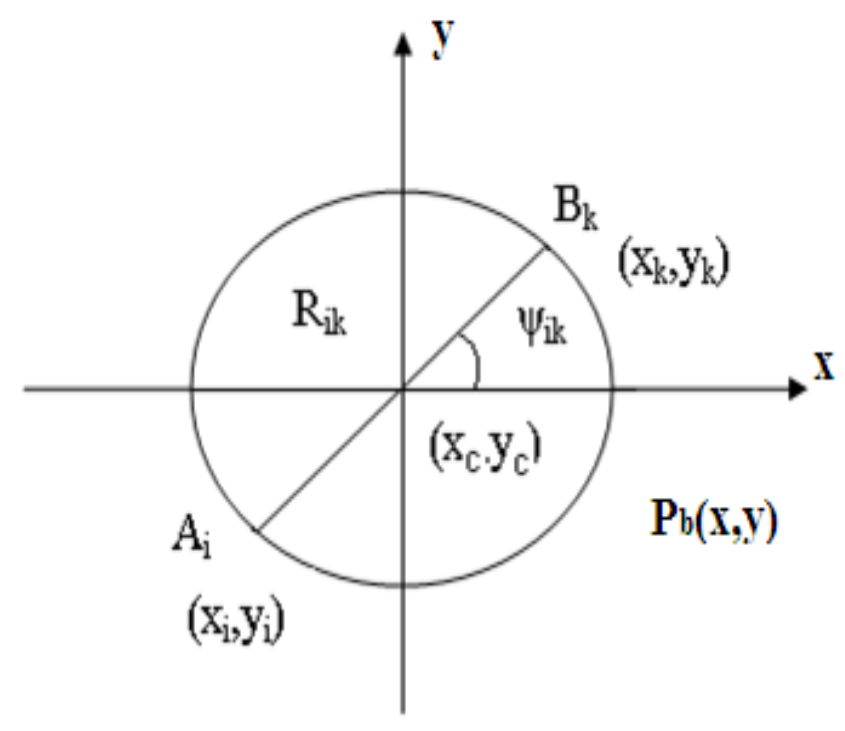

Figure 1. Central image chords determination.

In this case, the object's image can be characterized by the function $H\left(\psi_{\mathrm{ik}}, R_{\mathrm{ik}}\right)$, which describes all possible chords drawn through the point $\left(\mathrm{x}_{\mathrm{c}}, \mathrm{y}_{\mathrm{c}}\right)$. Transformation, through which was obtained the function $\mathrm{H}\left(\Psi_{\mathrm{ik}}, \mathrm{R}_{\mathrm{ik}}\right)$ was named the Central Image Chord Transformation (CICT) [16]:

$$
\mathrm{P}_{\mathrm{b}}(\mathrm{x}, \mathrm{y}) \rightarrow \mathrm{T}\left\{\mathrm{P}_{\mathrm{b}}(\mathrm{x}, \mathrm{y})\right\}=\mathrm{H}\left(\Psi_{\mathrm{ik}}, \mathrm{R}_{\mathrm{ik}}\right)=\mathrm{H}\left(\mathrm{x}_{1}, \mathrm{y}_{1}\right)=\mathrm{P}_{\mathrm{b}}\left(\mathrm{x}_{1}, \mathrm{y}_{1}\right),
$$

where $\mathrm{T}\{\ldots\}$ - the operation of CICT,

$$
\mathrm{x}_{1}=\Psi_{\mathrm{ik},}, \mathrm{y}_{1}=\mathrm{R}_{\mathrm{ik}} .
$$

The Logarithmic Central Image Chord Transformation (LCICT) [16] is realizing by taking the logarithm of the parameter $\mathrm{R}_{\mathrm{ik}}$ of the function $\mathrm{H}\left(\Psi_{\mathrm{ik}}, \mathrm{R}_{\mathrm{ik}}\right)$ :

$$
\operatorname{LT}\left\{H\left(\Psi_{\mathrm{ik}}, \mathrm{R}_{\mathrm{ik}}\right)\right\}=\mathrm{LH}\left(\Psi_{\mathrm{ik}}, \ln R_{\mathrm{ik}}\right)=\mathrm{LH}\left(\Psi_{\mathrm{ik}}, \mathrm{W}_{\mathrm{ik}}\right)=\mathrm{P}_{\mathrm{b}}\left(\mathrm{x}_{2}, \mathrm{y}_{2}\right) \text {, }
$$

where $L T\{\ldots\}$ - the operation of LCIHT,

$$
\mathrm{x}_{2}=\Psi_{\mathrm{ik}}, \mathrm{y}_{2}=\mathrm{W}_{\mathrm{ik}}=\ln \mathrm{R}_{\mathrm{ik}}
$$


As was shown in [16], the rotation of the initial image will shift the function $R(\Psi)$ along the axis $\psi$, i.e. the CICT operation allows to achieve invariance to the rotation of the object. In the case of the LCICT transformation, the object scale change will shift the function $\ln R(\Psi)$ along the axis $L n R$, i.e. this transformation allows to achieve the invariance to the scale change. The described operations of CICT and LCICT can be realized in the software or digital signal processors, as well as in the specialized optical processors.

\section{System for invariant object recognition and space parameters determination}

Based on the described in section 2 image chords transformation operations, the system for invariant object recognition and space parameters determination (position, scale, and angular orientation) was developed (Figure 2).

The system contains a laser 1 , an image input module 2, an optical multiplier 3, a processor 4 for calculating the object's position, processors 5 and 9 for image coordinate transformation, an optical processor 6 for implementing CICT/LCICT operations, a processor 7 for control and data processing, a processor 8 for Fourier transformation and calculating the image's Fourier spectrum maximum frequency and complexity, and processor 10 for object angular orientation determination.

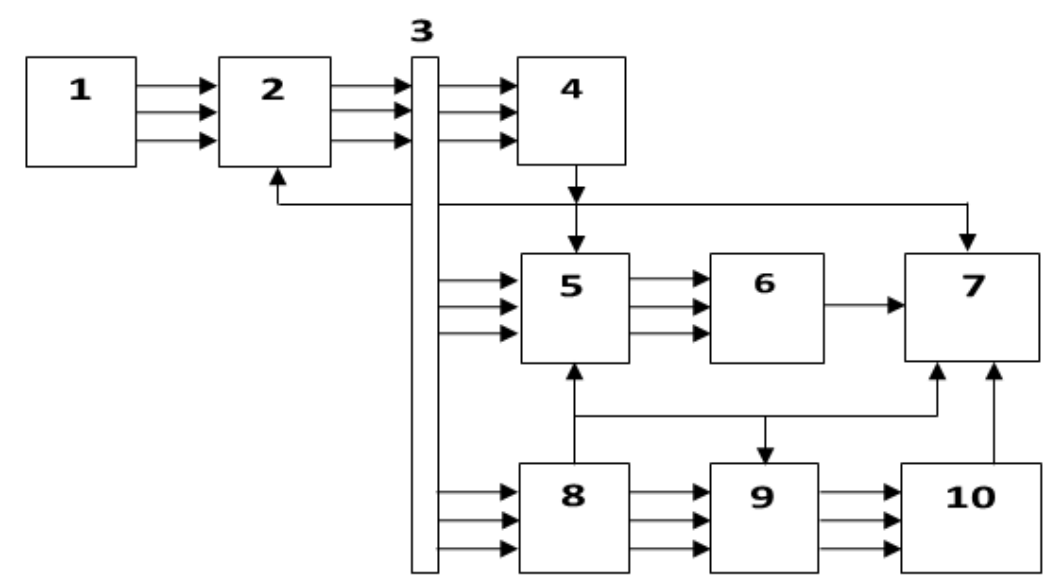

Figure 2. The system for object recognition and parameters determination.

Let the object's image is described by function $P\left(x^{\prime}, y^{\prime}\right)=P\left(x+e_{3}, y+e_{4}, e_{1}, e_{2}\right)$, where $e_{1}$ and $e_{2}$ are the object's scale and rotation changes respectively, and $e_{3}$ and $e_{4}$ are the object's displacements on the axes $x$ and $y$. The system functions as follows. The image $P\left(x^{\prime}, y^{\prime}\right)$ is introduced in module 2 . The light beam from laser 1 passing through module 2 is modulated in intensity by the function $P\left(x^{\prime}, y^{\prime}\right)$ and then is divided into three identical light beams by multiplier 3 . The first light beam is fed to processor 4 , where the position of the object in the plane (parameters $e_{3}, e_{4}$ ) is determined. At the same time, in processor 8 the Fourier spectrum of the input image is formed:

$$
P\left(x^{\prime}, y^{\prime}\right) \rightarrow F T\left\{P\left(x^{\prime} y^{\prime}\right)\right\}=P\left(u^{\prime} v^{\prime}\right)
$$

The maximum frequency $f_{m}$ of the Fourier spectrum $P\left(u^{\prime}, v^{\prime}\right)$ and the complexity $S L$ of image $P\left(x^{\prime}, y^{\prime}\right)$ are calculated in this processor. The function $P\left(u^{\prime}, v^{\prime}\right)$ then is introduced into processor 9 . The data regarding parameters $e_{3}, e_{4}$, and $S L$ are introduced into processors 5 , about $S L$ - into processor 9 , and regarding $e_{3}, e_{4}$, and $f_{m}$ - into processor 7 . In the processor 5 , based on the parameters $e_{3}, e_{4}$ and $S L$, the function $P\left(x^{\prime}, y^{\prime}\right)$ is centered: 


$$
P\left(x^{\prime}, y^{\prime}\right) \rightarrow P\left(x_{1}{ }^{\prime}, y_{1}{ }^{\prime}\right)=P\left(x_{1}, y_{1}, e_{1}, e_{2}\right) \text {. }
$$

Then, the function $\mathrm{P}\left(\mathrm{x}_{1}^{\prime}, \mathrm{y}_{1}^{\prime}\right)$ is input into processor 6 , where the operation of chords transformation is performed and a feature vector $\mathbf{v}$ is formed. The data from processor 6 are sent to processor 7 , where the object is classified and the scale value $e_{1}$ is determined based on the $f_{m}$ value. The object classification algorithm realized in processor 7 is based on the Fisher criterion [17] and suppose the forming of a linear discrimination function using sets of reference images of different classes at different angular orientations, scales, etc.

The value of parameter $e_{1}$ is sent from processor 7 to processor 9 , where the function $\mathrm{P}\left(\mathrm{u}^{\prime}, \mathrm{v}^{\prime}\right)$ is normalized on $\mathrm{e}_{1}$ and converted to a polar coordinate system:

$$
P\left(u^{\prime}, v^{\prime}\right)=P\left(u, v, e_{1}, e_{2}\right) \rightarrow P\left(u_{1}^{\prime}, v_{1}^{\prime}\right)=P\left\{u_{1}+u_{0}\left(e_{2}\right), v_{1}\right\},
$$

where $u^{\prime}{ }_{1}=\operatorname{arctg}(v / u), v^{\prime}{ }_{1}=\left(u^{2}+v^{2}\right)^{1 / 2}$

Wherein, the effect of the object rotation (parameter $e_{2}$ ) is reduced to a shift of the function $P\left(u_{1}^{\prime}, v_{1}\right)$ along the axis $u_{1}$. This function $P\left(u_{1}^{\prime}, v_{1}^{\prime}\right)$ is sent from processor 9 to processor 10 , where the parameter value $e_{2}$ is calculated based on the component $u_{0}\left(e_{2}\right)$ by faze extraction [18].

\section{The model of data processing flow in the system}

Based on the system's functioning description, let determine the stages of data processing as $\mathrm{Si} / \mathrm{j}$, where "i" is the stage nr. and " $\mathrm{j}$ " - is the nr. of the system's unit, where the corresponding stage is realizing. In this case, stage $\mathrm{S} 1 / 2$ will consist in image $\mathrm{P}\left(\mathrm{x}^{\prime}, \mathrm{y}^{\prime}\right)$ input into module $2 ; \mathrm{S} 2 / 4$ - object's position parameters $\mathrm{e}_{3}, \mathrm{e}_{4}$ determination in processor 4; S3/8 - image's Fourier spectrum maximum frequency $\mathrm{f}_{\mathrm{m}}$ and complexity $\mathrm{SL}$ determination in processor 8; S4/5 - object's image centering in processor 5; S5/6 - image chord transformation in processor 6; S6/7 - object classification in processor 7; S7/7 - object's scale $\mathrm{e}_{1}$ determination in processor $7 ; \mathrm{S} 8 / 9$ - object image Fourier spectrum normalization on parameter $\mathrm{e}_{1}$ in processor 9 , and $\mathrm{S} 9 / 10$ - object's rotation $\mathrm{e}_{2}$ determination in processor 10.

Figure 3 presents the model of data processing flow in the system, from which we can establish, that stages S2/4 and S3/8 of data processing can be realized in parallel in processors 4 and 8 respectively. Every processing stage will be characterized by respective processing time, which will permit to determine the system's processing time and productivity.

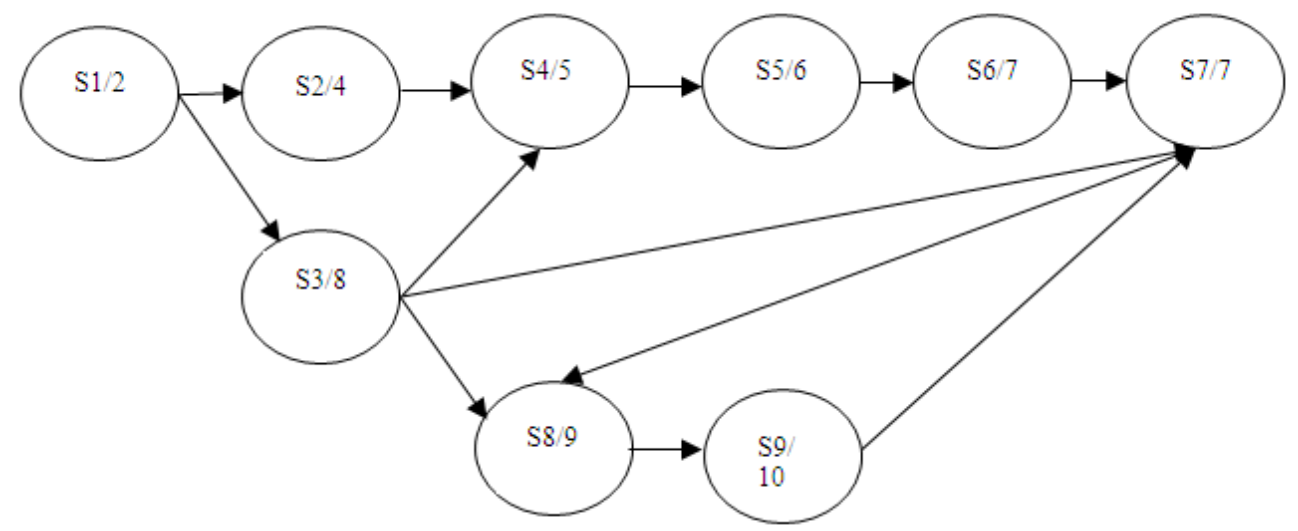

Figure 3. The model of data processing flow in the system. 


\section{Estimation of system's data processing time and throughput}

The time of data processing in the system will be determined based on the algorithm of its functioning described in sections 4 and 5 :

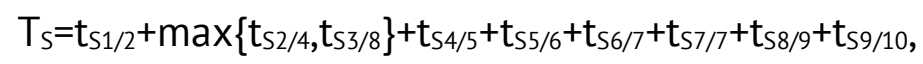

were $t_{s}$ is the stage's data processing time.

The time $t_{s 1 / 2}$ of the image input in module 2 will be determined by the parameters of the electro-optical spatial light modulator, $t_{s 1 / 2}=0.1 \mu s$ [19]. The $t_{s 2 / 4}$ is the time of the object's position $e_{3}, e_{4}$ determination in processor $4, t_{s 2 / 4}=1.01 \mu s$ [20]. The $t_{s 3 / 8}$ is the time of the Fourier spectrum maximum frequency $\mathrm{f}_{\mathrm{m}}$ and complexity SL determination in processor $8, t_{s 3 / 8}=4.24 \mu s$ [21]. The $t_{s 4 / 5}$ is the time of the object's image centering in processor 5 . The time spent at this stage will be as follows [22]: $t_{s 4 / 5}=14.2+\alpha^{2} G_{n}\left(t_{e p}+\alpha_{h} t_{t p}\right)$, where the parameter $\alpha_{s}$ characterizes the complexity of the input image; $G_{n}$ - the total number of image pixels, $G_{n}=N_{n} \times M_{n} ; \alpha_{h}$ - determines the part of highly informative pixels in the image; $t_{e p}, t_{t p}$ - times of the pixel's extracting from the image and converting its coordinates. At $t_{\mathrm{ep}}=0.015 \mu \mathrm{s}$ and $\mathrm{t}_{\mathrm{tp}}=0.28 \mu \mathrm{s}$,

$$
t_{s 4 / 5}=14.2+\alpha^{2} G_{n}\left(0.015+0.28 \alpha_{h}\right), \mu s .
$$

Figure 4 presents the results of $t_{54 / 5}$ calculation which show that at the increase of the image complexity $\alpha_{s}$ from 0,025 to 1 , of the number of image pixels $G_{n}$ from $64^{2}$ until $512^{2}$ and $\alpha_{h}=0.1$, the value $t_{s 4 / 5}$ increase from $14.31 \mu$ s until $11.28 \mathrm{~ms}$.

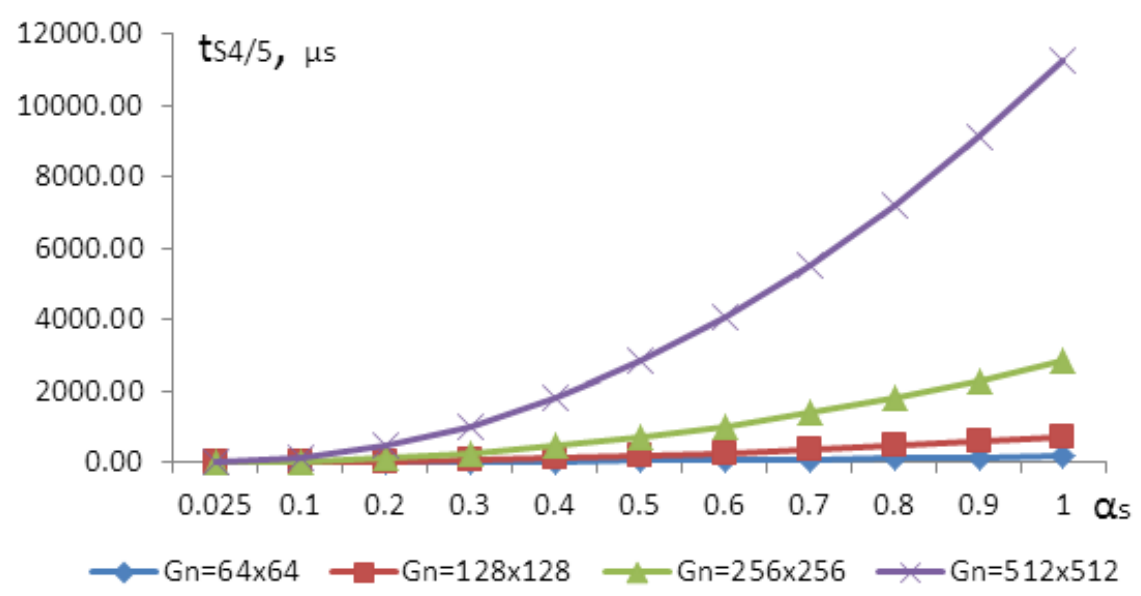

Figure 4. The time of the image normalization.

The time of the image chord transformation operation in processor 6 can be determined as $t_{55 / 6}=t_{m o}+t_{p d}[16]$, where $t_{m o}$ is the time of the image binarization operation by the matrix of threshold optrons, and $t_{p d}$ is the time of image scanning by a photodetector. At $\mathrm{t}_{\mathrm{mo}}=\mathrm{t}_{\mathrm{pd}}=0.1 \mu \mathrm{s}, \mathrm{t}_{\mathrm{s} 5 / 6}=0.2 \mu \mathrm{s}$.

The $t_{56 / 7}$ is the time of the object classification in processor 7. At the identification of the $m_{k}$ classes' objects, the time spent will be as follows: $t_{s 6 / 7}=\left(m_{k}-1\right)\left[D P\left(t_{y}+t_{c}\right)\right]$, where $D P$ is the object's image feature vector $\mathbf{v}$ length; $t_{y}, t_{c}$ - the time of multiplication and addition operations, respectively. At $t_{y}=0.04 \mu s$ and $t_{c}=0.02 \mu s$, 


$$
\mathrm{t}_{56 / 7}=0.06\left(\mathrm{~m}_{\mathrm{k}}-1\right) \mathrm{DP}, \mu \mathrm{s} \text {. }
$$

Figure 5 presents the results of $\mathrm{t}_{56 / 7}$ calculation, which shows that at the increase of the object classes number $m_{k}$ from 2 to 10 and object's image feature vector $\mathbf{v}$ length DP from 180 to 720 , the value $t_{56 / 7}$ increase from $10.8 \mu$ s until $388.8 \mu$ s.

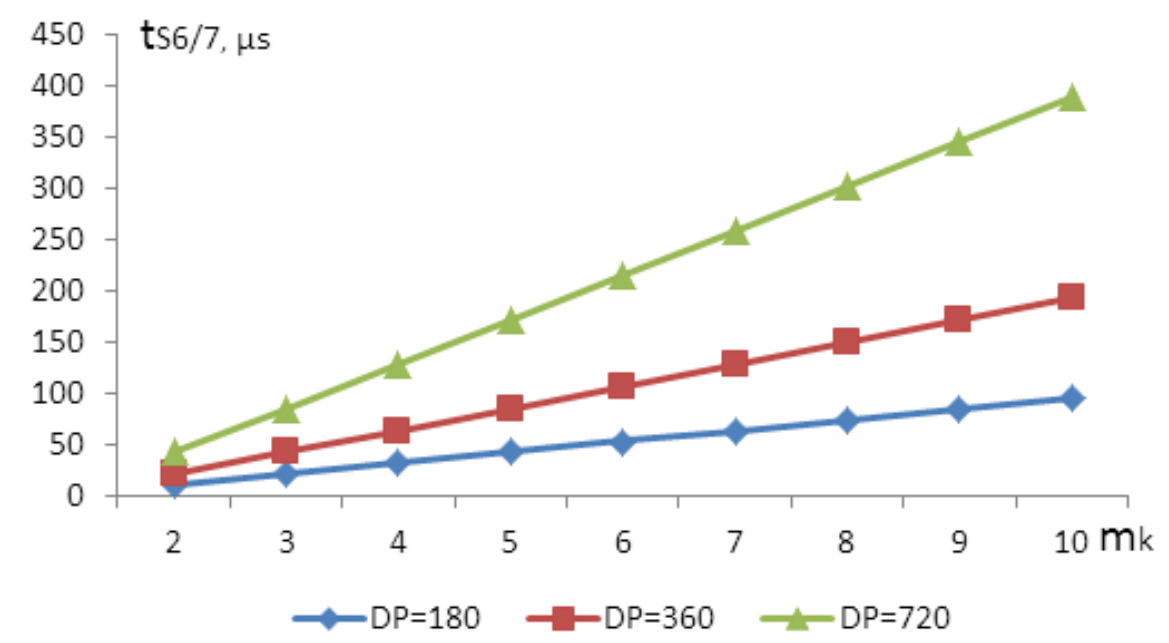

Figure 5. Time of the object classification.

The $t_{57 / 7}$ is the time of the object's scale $e_{1}$ determination in processor $7, t_{57 / 7}=0.04 \mu \mathrm{s}$ [16]. The $t_{s 8 / 9}$ is the time of the object image Fourier spectrum normalization on the parameter $e_{1}$ in processor $9, \mathrm{t}_{58 / 9}=\mathrm{t}_{54 / 5}$.

The $t_{59 / 10}$ is the time of the object's rotation $e_{2}$ determination in processor 10 . The value of the parameter $t_{59 / 10}=15.1+N_{n}\left(0.8 N_{n}+5.15\right), \mu s$ [18]. At the increasing the $N_{n}$ from 64 until 512 , the value of $\mathrm{t}_{59 / 10}$ increases from $3,6 \mathrm{~ms}$ until $212,4 \mathrm{~ms}$.

Taking into account that $\mathrm{t}_{53 / 8}>\mathrm{t}_{52 / 4}$, the data processing time in the system can be determined as:

$\mathrm{T}_{\mathrm{s}}=\mathrm{t}_{51 / 2}+\mathrm{t}_{53 / 8}+\mathrm{t}_{54 / 5}+\mathrm{t}_{55 / 6}+\mathrm{t}_{56 / 7}+\mathrm{t}_{57 / 7}+\mathrm{t}_{58 / 9}+\mathrm{t}_{59 / 10}$.

After substitution of the $t_{s i j}$ values,

$T_{s}=48.08+N_{n}\left\{N_{n}\left[2 \alpha_{s}^{2}\left(0.015+0.28 \alpha_{n}\right)+0.8\right]+5.15\right\}+0.06\left(m_{k}-1\right) D P, \mu s$.

Figure 6 presents the data regarding processing time on different stages for $\mathrm{N}_{\mathrm{n}}=256$, $\mathrm{DP}=180, \mathrm{~m}_{\mathrm{k}}=10 \mathrm{a}_{\mathrm{s}}=0.5$. In this case, the data processing time in the system will be $\mathrm{T}_{\mathrm{s}}=55.24 \mathrm{~ms}$. These data show that maximal processing time is on the stage $\mathrm{S} 9 / 10$, $\mathrm{t}_{59 / 10}=53.7 \mathrm{~ms}$. The throughput of the system will be $\mathrm{TP}_{\mathrm{s}}=\mathrm{ICP} / \mathrm{t}_{59 / 10}$, where ICP is the input image capacity. At ICP $=256 \times 256 \times 8$ and $t_{59 / 10}=53.7 \mathrm{~ms}$, the value of $\mathrm{TP}_{\mathrm{s}}=9.7 \times 10^{6} \mathrm{bits} / \mathrm{sec}$.

\section{Optimized system}

From Figure 6 is evident that the system is not optimal from the throughput point of view, because the processing time on stage $59 / 10$ is much bigger than on other stages. To obtain an optimal system, it is necessary to reduce the processing time on this stage until processing time on the stages S4 and S8 by using other kinds of processors, introducing supplementary parallelism, etc. [23]. At the $\mathrm{t}_{\mathrm{s} 9 / 10}=\mathrm{t}_{\mathrm{s} / 9}$, the throughput of the optimized system will increase up to $\mathrm{TP}_{\mathrm{so}}=0.73 \times 10^{\circ} \mathrm{bits} / \mathrm{sec}$, or, on $\mathrm{k}=\mathrm{TP}_{\mathrm{so}} / \mathrm{TP}_{\mathrm{s}}=75$ times. 
For optimized system, the total data processing time will be: $\mathrm{T}_{\mathrm{so}}=$ $\mathrm{t}_{\mathrm{s} 1 / 2}+\mathrm{t}_{\mathrm{s} 3 / 8}+\mathrm{t}_{\mathrm{s} 4 / 5}+\mathrm{t}_{\mathrm{s} / 6}+\mathrm{t}_{\mathrm{s} 6 / 7}+\mathrm{t}_{\mathrm{s} 7 / 7}+\mathrm{t}_{\mathrm{s} 8 / 9}+\mathrm{t}_{\mathrm{s} 8 / 9}=2.25 \mathrm{~ms}$, or, on $\mathrm{w}=\mathrm{T}_{\mathrm{S}} / \mathrm{T}_{\mathrm{SO}}=24.5$ times less in comparison with initial system.

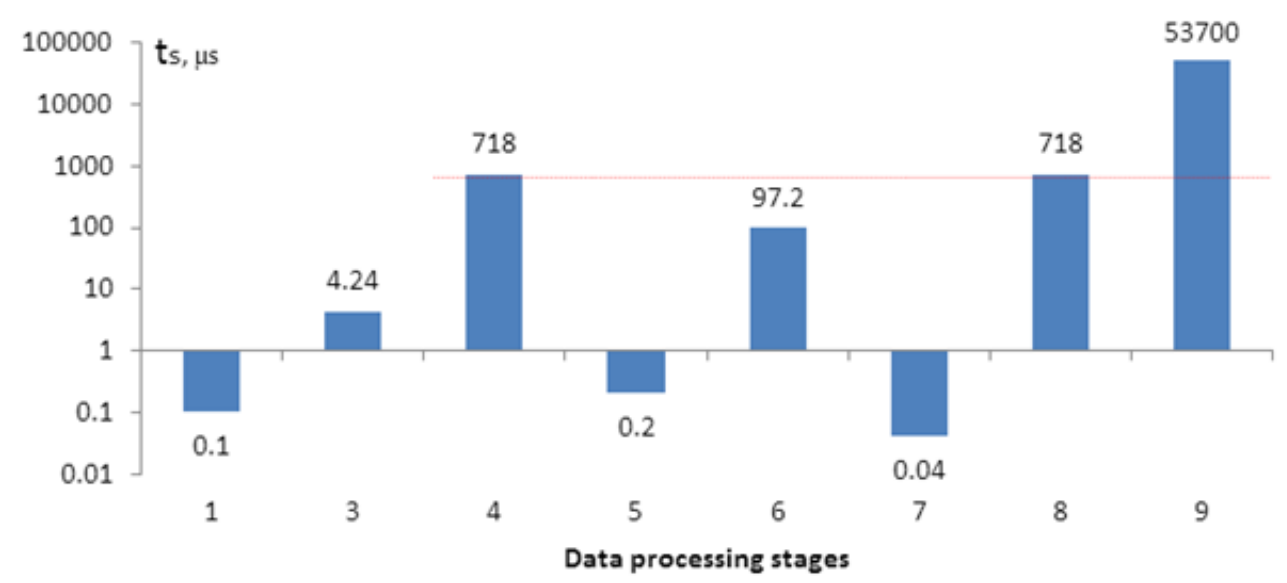

Figure 6. Data processing time on different stages in the system.

\section{Conclusions}

Two types of image transformations are described - the Central and Logarithmic Central Image Chords Transformations (CICT and LCICT) which allows achieving invariance to the displacement and rotation of the object in the case of CICT, and the LCICT operation - to the displacement, rotation, and scale at LCICT operation realization. These transformations permit applying high-speed algorithms and techniques at the stage of object classification, significantly reducing the volume of computational operations and implementing a real-time mode in image analysis.

Based on the proposed algorithms of image chords transformation, the structure of an optical-electronic system for invariant object recognition is developed. The system is of a multiprocessor functionally distributed architecture, in which various modules implement separate image processing operations. The system allows to recognize objects regardless of their position, scale, or rotation, and to determine the parameters for targeting.

The system provides the organization of adaptive information processing depending on the complexity of input images at the stages of classification and image coordinates transformation, which can significantly improve the process of image analysis and decisionmaking processes. The model of a data processing flow in the system was elaborated that permits the determination of the stages of signal processing which can be realized in parallel. An analytical performance assessment of the image processing operations and the system was made, which permitted to determine the data processing stage which influences the system's throughput. The approach of the system's optimization was applied, which allow to decrease of the total data processing time by 24.5 times and to increase the system's throughput by 75 times.

\section{References}

1. Jin L., Qinzhang Y., Guibin C. Infrared ship target detection based on the combination of Bayesian theory and SVM. In: Proc. SPIE 11429, 2019.

2. Ciocoiu I. B. Invariant pattern recognition using support vector data description and tangent distance. In: Proc. of the Romanian Academy, Ser. A, Vol. 20, Nr. 2, pp. 197-204, 2019.

3. Uchaev D., Malinnikov V. Spectral-spatial classification of hyperspectral images based on multifractal features. In: Proc. SPIE 11533, 2020. 
4. Senlin Y., Jing S., Yingni D. Dangerous object detection by deep learning of convolutional neural network. In: Proc. SPIE 11427, 2020.

5. Garcia-Salgado B., Ponomaryov V. Parallel artificial neural networks using wavelet-based features for classification of remote-sensing hyperspectral images. In: Proc. SPIE 11401, 2020.

6. Hao D., Qianqian D., Jiaxing M. Object detection based on hierarchical visual perception mechanism. In: Proc. SPIE 11429, 2019.

7. Tuama A., Abdulrahman H., Magnier B. Integrated convolutional neural network model with statistical moments layer for vehicle classification. In: Proc. SPIE 11433, 2019.

8. Benouini R., Batioua I., Zenkouar K. Fractional-order orthogonal Chebyshev moments and moment Invariants for image representation and pattern recognition. In: Pattern Recognition, Vol. 86, February, pp. 332-343, 2019.

9. Spiliotis I., Karampasis N., Boutalis Y. Fast computation of Hahn moments on gray images using block representation. In: Journal of Electronic Imaging, Vol. 29, Issue 1, 2020.

10. Danyu S., Jiangming K., Songnan C. Color-based moment invariants under changing illumination. In: Journal of Electronic Imaging, Vol. 29, Issue 2, 2020.

11. Hoang T., Tabbone S. Invariant pattern recognition using the RFM descriptor. In: Pattern Recognition, Vol.45, Issue 1, January, pp.271-284, 2012.

12. Chen G., Xie W. Invariant pattern recognition using ring-projection and dual-tree complex wavelets. In: Proc ICWAPR International Conference on Wavelet Analysis and Pattern Recognition, pp. 182 - 186, 2011.

13. Fernández A. Optical architectures for pattern recognition with the generalized Hough transform. In: Proc. SPIE 11351, 2020.

14. Nichol D.G. Hybrid optical digital computation of global chord functions. In: Optics Communications, Vol.43, Issue 3, October, pp. 168-170, 1982.

15. Casasent D., Chang W. Generalized chord transformation for distortion - invariant optical pattern recognition. In: Applied Optics, Vol. 22, nr.14, pp. 2087-2094, 1983.

16. Perju V., Cojuhari V. Central and logarithmic central image chord transformations for invariant object recognition. In: Journal of Engineering Science. 2021, Vol. XXVIII, no. 1 (2021), pp. 38 - 46 ISSN 25873474/E-ISSN 2587-3482. https://doi.org/10.52326/jes.utm.2021.28(1).03.

17. Duda R. O., Hart P. E., Stork D. G. Pattern Classification. Wiley Interscience, 2000.

18. Katys G.P., Perju V.L., Rotary S.L. Methods and computer means of image processing. Shtiintsa, Kishinev, 1991.

19. Perju V., Casasent D. Optical multichannel correlators for high-speed targets detection, recognition, and localization. In: Proc. SPIE 8398, 2012.

20. Perju V.L., Casasent D.P., Young R., Perju V.V. Optical correlation processors for high-speed objects recognition and localization. In: Proc. Intern. Conf. Telecommunications, Electronics and Informatics. Chisinau, Moldova, May 18-19, 2012.

21. Perju V., Casasent D. The investigation of the Fourier spectrum-based image complexity metrics for recognition applications. In: Proc. SPIE 8398, 2012.

22. Perju V.L. The method of adaptive image coordinate transformation. In: Proc. SPIE 1978, pp. 280-288, 1993.

23. Perju V., Sofronescu I. High-Speed Targets Recognition Systems Design based on Optimal Combination of the Optical and Electronic Processors. In: Proc. ITSN-2017 International Conference on Information Technologies, Systems, and Networks, 17 - 18 Oct. 2017, pp. 306-324, Chisinau, ULIM, 2017. 